Article

\title{
Elution of Artificial Sputum from Swab by Rotating Magnetic Field-Induced Mechanical Impingement
}

\author{
Shubham Banik ${ }^{1}$, James Mahony ${ }^{2}$ and P. Ravi Selvaganapathy ${ }^{1, *}$ \\ 1 Department of Mechanical Engineering, School of Biomedical Engineering, McMaster University, \\ 1280 Main Street West, Hamilton, ON L8S 4L8, Canada; baniks2@mcmaster.ca \\ 2 Department of Pathology and Molecular Medicine, McMaster University, 1280 Main Street West, Hamilton, \\ ON L8S 4L8, Canada; mahonyj@mcmaster.ca \\ * Correspondence: selvaga@mcmaster.ca
}

Received: 2 October 2017; Accepted: 30 November 2017; Published: 3 December 2017

\begin{abstract}
Cotton-tipped applicator swabs are used as a collection device for many biological samples and its complete elution is a desired step for clinical and forensic diagnostics. Swabs are used to collect infectious body fluids, where the concentration of pathogens can range from $1 \times 10^{4} \mathrm{CFU} / \mathrm{mL}$ (colony forming units $/ \mathrm{mL}$ ) in respiratory-tract infections and $1 \times 10^{5}$ in urinary-tract infections, to up to $1 \times 10^{9} \mathrm{CFU} / \mathrm{mL}$ in salivary samples. These samples are then eluted and lysed, prior to DNA (De-oxy Ribonucleic Acid) analysis. The recovery of micro-organisms from a matrix of swab fibres depends on the nature of the body fluid, the type of the swab fibres, and the process of elution. Various methods to elute samples from swab include chemical digestion of fibres ( $20 \%$ recovery), centrifugation ( $\sim 58 \%$ recovery), piezoelectric vibration, or pressurized fluid-flow ( $\sim 60 \%$ recovery). This study reports a magnetically-actuated physical impingement method for elution and recovery of artificial sputum samples from cotton fibres. A device has been fabricated to induce a rotating magnetic field on smaller magnetic particles in a vial that strikes the swab within a confined gap. Elution from the swab in this device was characterized using 2\% Methyl cellulose in deionised water, loaded with fluorescent-tagged polystyrene beads and E. coli at various concentrations. The recovery efficiency was found to increase with both rotational speed and elution time, but plateaus after 400 RPM (Revolutions per minute) and $120 \mathrm{~s}$, respectively. At a higher concentration of polystyrene beads $\left(5 \times 10^{8}\right.$ particles $\left./ \mathrm{mL}\right)$, a maximum recovery of $\sim 85 \%$ was achieved. With lower concentration, $\left(1 \times 10^{5}\right.$ particles $\left./ \mathrm{mL}\right)$ the maximum efficiency $(\sim 92.8 \%)$ was found to be almost twice of passive elution (46.7\%). In the case of $E$. coli, the corresponding recovery efficiency at $3.35 \times 10^{5} \mathrm{CFU} / \mathrm{mL}$ is $90.4 \%$ at $500 \mathrm{RPM}$ and $120 \mathrm{~s}$. This elution method is expected to have a wide applicability in clinical diagnostics.
\end{abstract}

Keywords: swab; elution; magnetic field; recovery efficiency; impingement

\section{Introduction}

Sampling and elution are the very first steps in the preparation of biochemical assays, which are used in the clinical testing or analysis of environmental samples. Swabs are widely used to collect biological samples, such as saliva, nasal sputum, semen, blood, or urine from the human body. They are made of cotton, rayon, polyurethane foam, or polyester, and are available in a spun or flocked tipped format. Cotton based collection methods are the most widely used sample collection techniques with salivary or sputum based immunoassays because of its easy availability, low cost and high absorbance. Apart from biological samples, swabbing techniques are also used for DNA analysis of touched evidence [1], recovery of spores from environmental surfaces [2,3], or the removal of dried blood stains [4]. Depending on the field of application, the type of swab used could vary (dry and 
pre-moistened swabs [1], or macrofoam swabs [2]). Recovery of samples from these swab tips requires a stable and high efficiency elution technique, and the success rate of all the downstream processes, like lysis and DNA amplification, are governed by the number of captured and lysed cell.

The concentration of microbial cells in body fluids has a significant influence on diagnostic sensitivity of any assay. The concentration of pathogens can range from $1 \times 10^{4} \mathrm{CFU} / \mathrm{mL}$ in nasal sputum samples of nosocomial pneumonia patients [5] to $1.6 \times 10^{9} \mathrm{CFU} / \mathrm{mL}$ in the saliva of periodontitis patients [6]. Apart from these common body fluids, samples from blood, vaginal fluid, urinary tracts, or semen are also collected by swabs [7,8]. Sperm cell recovery is a critical for elution in sexual assault samples of forensic cases. Recovery efficiency of spermatozoa, as low as $10 \%$ [8], collected from a victim's body hinders the subsequent process of lysis and PCR amplification, thereby incorrectly characterizing the sample as negative to sperms. Higher recovery is essential for cases where trace amounts of bacterial cells are available in the body fluids.

Several methods have been used to increase the recovery of the sample constituents from the swab. Among them, chemically enhanced recovery [8-10] and water extraction techniques involving vortexing $[2,3]$ are the most common techniques for elution and recovery of sperm cells. Enzymatic dissolution of cotton fibres result in 18-23\% recovery [8], which could be increased when detergents like Sarkosyl (54.4\%) and sodium dodecyl sulfate (SDS) (78.50\%) were used [9]. Vortexing can be used for the recovery of bacterial spores in environmental samples from cotton, macrofoam, polyester, and rayon swabs, with an average recovery of $6.6 \%$ and $26.7 \%$ for dry and pre-moistened swabs, respectively [3]. Sometimes, convective flow has been associated with vortexing to enhance the recovery $(41.7 \%)$ of Bacillus anthracis spores [2,3]. A comparative performance by Lutz et al. [11] for extraction of Staphylococcus aureus from metal surfaces with abrasive techniques showed an apparent recovery efficiency of $18 \%$ for electrostatic wipes, $10 \%$ for roller sampler and $0.04 \%$ for contact plates, which are lesser than those that were obtained from ultrasonic elution processes (32.1\%) [12-14]. Another widely used process is the thermal elution technique $[13,15,16]$, which possess the added advantage of eluting and lysing the cells simultaneously. Mechanical and magnotephoretic agitation methods require higher volumes or a continuous flow of eluent and highly intricate systems for detecting the target analyte to yield a maximum of only $55 \%$ recovery.

Several swabbing devices have also been reported in the patent literature. These devices use periodic vibration or compressible forces for elution. For example, a swab analyser with a porous swabbing pen was designed by Wuske et al. [17], which utilises $5 \mathrm{~mL}$ of eluent flow for the removal of immunological components taken from skin and pharyngeal cavity. Pelssers et al. [18] provided a compressible device for the elution of the receiving end of swabs, which allows for a conditioning fluid to be flown into the elution zone from a blister along a transfer path. Piezolelectric methods [19] allow for an actuator to strike a flexible film at low frequency ranges between 10 and $100 \mathrm{~Hz}$, but also require a flow of conditioning fluid across the swab. Nason [20] used one or more test fluids to be delivered to the tip of the swab through the shank after being squeezed out of porous filter membranes with a hydrophobic sealant. Alternating magnetic fields [21] have also been used for extraction of analytes from samples by tagging antibodies with magnetic beads, which binds to antigens on bacteria and gets removed on the influence of the magnetic trap.

Similar issues of low recovery are also encountered in sample processing for immunoassays in clinical diagnostics. Rose et al. [3] confirmed the preference of vortexing (43.6\%) over sonication (17.7\%) as a better technique of elution of bacterial cells from pre-moistened cotton swabs. Higher recovery efficiencies upto $55 \%$ can be obtained with vortexing, while tangential shear forces with associated flow (piezoelectric, pressurized flow) can yield a maximum of $60 \%$ recovery. Different extraction techniques by vortexing, squeezing, pummelling, maceration, rolling, and differential extraction of semen from swabs were compared by Allard et al. [22]. This study showed higher average elution times ( $\sim 5 \mathrm{~min}$ ) with $8000-13,000 \mathrm{RPM}$ and $\sim 400$ to $1000 \mu \mathrm{L}$ of eluate. Though chemical processes can provide higher elution efficiency $(\sim 78 \%)$, they require a complex combination of buffers, enzymes, and detergent solutions, and up to $2 \mathrm{~h}$ of incubation period, and it is also specific to the type of cells. 
In this paper, a mechanical technique of elution of cotton-tipped applicator swabs was developed that significantly improves the recovery efficiency. Here, we demonstrate the highest elution recovery procedure by inflicting physical impingement forces on the swab fibres by magnetic particles. The collection and release of polystyrene beads and E. coli bacteria are compared and characterized with physical parameters, such as rotational speed (RPM), elution time, and concentration. The ability of the device to elute micro-organisms like E. coli from artificial mucous samples has also been explored. Separate protocols for the quantification of particulates have been developed using microscopic pictures and fluorescent intensity measurements. It is expected that this method has broad applicability in sample elution of a variety of clinical and environmental samples that are collected using swabs.

\section{Working Principle and Device Design}

The elution device (Figure 1a,b) consists of a rotating mechanism, ring magnet, a vial at the center with the magnetic particles, and the electrical control unit. It resembles a sun and planetary gear mechanism, with a planetary gear rotating around the teeth of an external fixed gear, the centre distance between them being $38.1 \mathrm{~mm}$. The device is designed with 16 teeth on the planetary gear and a facewidth of $12 \mathrm{~mm}$ on both structures to prevent gear failure. The gear ratio between the mating gears is 2.9 , with a module of $2.5 \mathrm{~mm}$. A cylindrical vial ( $15 \mathrm{~mm}$ diameter and $45 \mathrm{~mm}$ height) is placed at the centre of the device, supported by clamps. Smaller magnetic particles $\left(3.2 \mathrm{~mm}^{3}\right)$ are placed in confinement (gap spacing $\sim 5 \mathrm{~mm}$ ) inside the walls of the vial. The planetary gear has slot for holding the diametrically magnetized ring/disk magnet. The smaller magnetic particles inside the vial get attracted by the magnet, which produces simultaneous rotation around its axis and revolution around the vial axis. The rotation of the magnet along its own axis is responsible for the physical impact on the fibres. The revolutionary trajectory allows for the particles to cover the entire surface area of the swab, thereby allowing maximum exposure. By adjusting the height of the vial, the magnetic particles can be levitated from the base, which allows them to cover the entire length of the swab in the vertical direction. Smooth transfer of rotational energy has been ensured by inserting snap rings below the bearings to prevent its fallout form position. A small beam supporting the planetary gear is also connected with the motor shaft that revolves around the central axis of the device. The device is connected to a DC power supply, which provides the necessary voltage for the DC motor (Figure 2a).

This device provides higher impact forces on the swab fibres, desired to improve elution efficiencies. In order to introduce multiple impingements and fluid circulation, a diametrically magnetized ring magnet is used and mechanically moved around the vial in a planetary fashion. The planetary rotation periodically changes the polarity of the magnet, which causes rotation of the magnetic particles in the fluid. The rotation of the particles is responsible for creating a scrubbing action to remove materials from the swab. The revolution of the ring magnet itself causes curvilinear translation of the magnetic particles around the swab. These particles strike the swab at different locations angles to provide the necessary force to elute the entrapped beads/cells. Simultaneous rotation and revolution of the particles is desired inside the vial to cover maximum surface area of the swab head. Repeated impingements along with turbulent motions of the fluid also allow for fresh fluid to enter the fibre matrix after each impact and dissolve the samples with the elution fluid.

The most important parameters that affect the recovery efficiency are the rotational speed of the magnet around the vial, the time of elution, and the concentration of the particles/cells in the sample. The rotational magnetic field is dependent on the speed of the motor. Higher rotational speed provides more number of impingements per minute, thereby facilitating higher removal of materials from the swab fibres. Hence, RPM can be identified as the most important parameter. Elution time reflects the amount of time that the impact forces are imparted to the swab and hence the trend in recovery should also influence the elution time. Concentration of the particulates in the sample is another important criterion that shows the number of eluted particles and chances of entrapment of the organisms in the fibrous matrix. The effect of these parameters needs to be characterized in order to determine the optimal operating conditions for high recovery efficiency. 


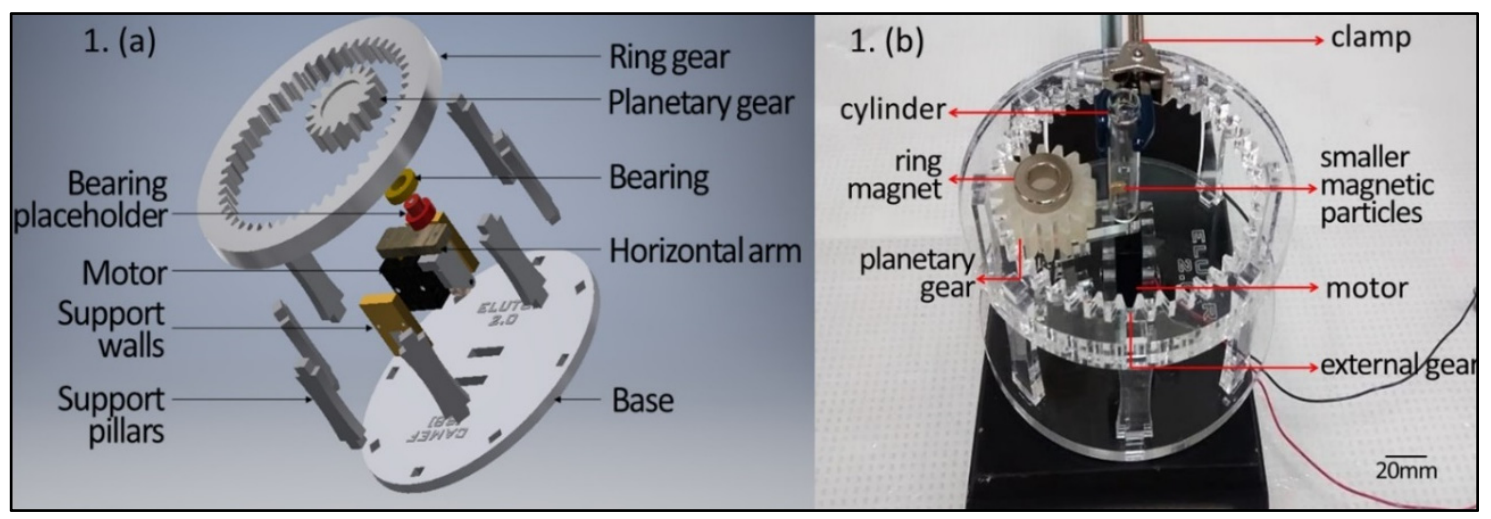

Figure 1. (a) Schematic of the device showing an exploded view of the various parts; (b) Fabricated and assembled device.

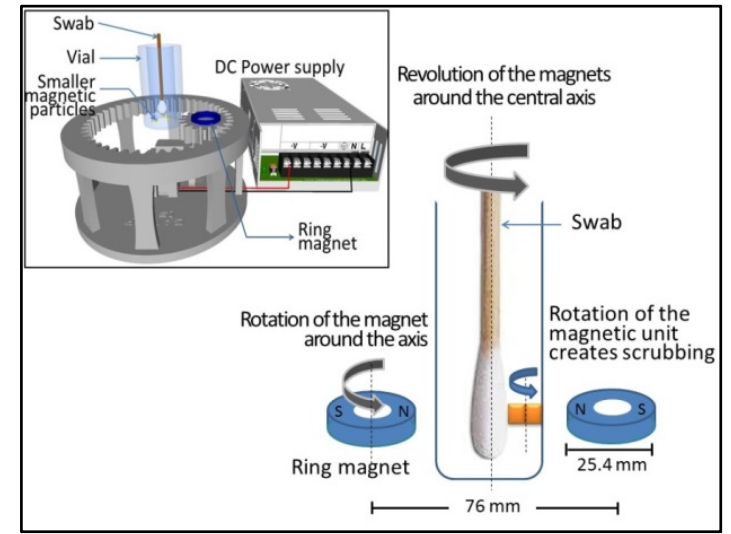

(a)

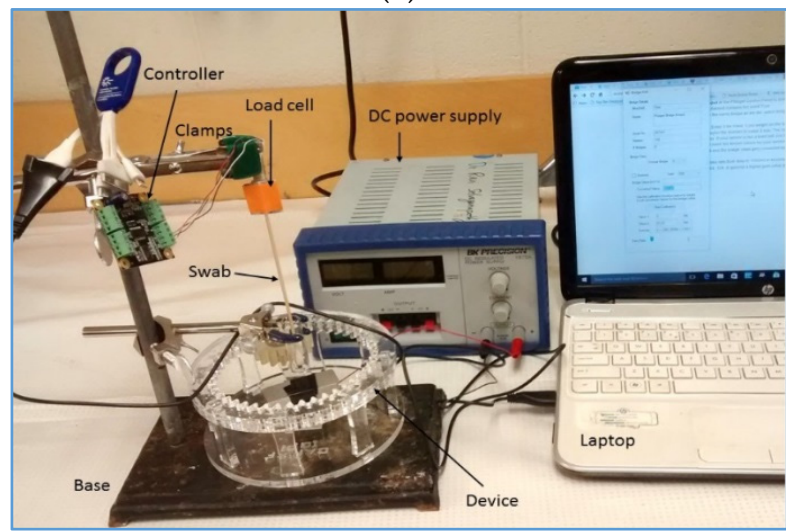

(b)

Figure 2. (a) Working forces on swab by impingement with smaller particles, actuated by the rotating ring magnet. (Inset) Experimental setup; (b) Experimental setup for measuring forces with load cell at the fixed end. The forces on the swab tip were then calculated by the principle of a cantilever beam, fixed at the measuring end.

\section{Materials and Methods}

\subsection{Materials}

Cotton-tipped applicators (manufactured by Medline Industries, Inc., Mundelein, IL, USA) were used to mimic nasal or mouth swabbing of a patient with a denser than normal sputum. Each cotton 
tipped applicators were $15.2 \pm 0.4 \mathrm{~cm}$ in length, with a swab tip length of $1.5 \pm 0.1 \mathrm{~cm}$. The shank of the swab is wooden, while the tip is latex-free cotton. Water absorptivity is an important parameter to define the physical property of swab fibres, which incorporates only a fraction of the total weight of the swab. The artificial sputum that was used in the set of experiments follows the properties of mucoid type of macroscopic sputum with a viscosity of $4.20 \pm 0.4$ poise [23] at a shear rate of $90 \mathrm{~s}^{-1}$. To obtain the desired sample, a $\%(\mathrm{w} / \mathrm{w})$ solution of Methyl Cellulose in deionized (DI) water was used. Negatively charge-stabilized colloidal polystyrene particles (Invitrogen FluoSpheres Carboxylate-Modified Microspheres, Fluorescent tagged) were used to replicate the presence of microbes in sputum. These polystyrene particles have a nominal bead diameter of $1.0 \mu \mathrm{m}$ and are labelled with orange fluorophore (proprietary dye) with an excitation/emission wavelength of $540 / 560 \mathrm{~nm}$. The microbeads are in an aqueous suspension of $1 \times 10^{10}$ particles $/ \mathrm{mL}$. They are mixed with the artificial sputum in varying ratios to mimic the microbe-infested sputum of a patient. To further validate the experiments with actual bacteria, Green Fluorescence Protein (GFP) tagged E. coli K12 (ATCC 27325, Excitation/emission wavelength: 487/509 nm) were used for the elution experiments. A ring magnet has been used to create a rotational magnetic field that imposes the smaller magnetic particles to provide the necessary kinetic energy of impact. The ring magnet is a N42 grade of diametrically opposite NdFeB magnet (K\&J Magnetics Inc., Pipersville, PA, USA), with dimensions of $1 / 2^{\prime \prime}(12.7 \mathrm{~mm})$ OD $\times 3 / 16^{\prime \prime}(4.76 \mathrm{~mm})$ ID $\times 1 / 4^{\prime \prime}(6.35 \mathrm{~mm})$ thick $\left( \pm 0.004^{\prime \prime} \times \pm 0.004^{\prime \prime} \times \pm 0.004^{\prime \prime}\right.$ $(0.1 \mathrm{~mm})$ tolerances). The ring magnet is strong enough to cause the levitation of the magnetic particles when the vial is placed in its position. The smaller magnetic particles are N42 grade of NdFeB block magnets with Ni-Cu-Ni (Nickel) plating, magnetized through thickness. These magnetic particles are cubical, with a dimension of $\left(1 / 8^{\prime \prime}\right.$ or $\left.3.175 \mathrm{~mm}^{3}\right)$. The rotation of the magnet by the motor produces a magnetic force on the particles under confinement (gap spacing $\sim 5.08 \mathrm{~mm}$ ) between the swab and the walls, repeatedly impinging on the swab at various angles and locations.

\subsection{Preparation of Samples}

Artificial sputum was prepared by dissolving methyl cellulose with DI water. Dry methyl cellulose, (Sigma Aldrich, St. Louis, MO, USA, product \# M0262) was first mixed with 1/3rd of the required volume of water, preheated to $80^{\circ} \mathrm{C}$, while being continuously stirred by a magnetic stirrer. The solution was continuously agitated until the methyl cellulose particles got evenly dispersed. The remainder of the water was added, and the temperature of the water was lowered to increase the solubility. The solution was stirred for another $30 \mathrm{~min}$. Thus, a required viscosity of $\sim 4.1$ poise at $20^{\circ} \mathrm{C}$ was obtained. The artificial sputum, which was used in the set of experiments, was formulated to mimic the properties of macroscopic mucoid type of natural sputum with a viscosity of $4.20 \pm 0.4$ poise [24] at shear rate of $90 \mathrm{~s}^{-1}$.

Fluorescent-tagged bacterial cells were used in counting the number of cells, when imaged under a fluorescent microscope. E. coli were cultured in Luria-Bertani (LB) broth by picking individual colonies form infested agar plates. They were kept overnight $(16 \mathrm{~h})$ in a shaking incubator at $37^{\circ} \mathrm{C}$ and 250 RPM, after being mixed with Ampicillin (1:1000 ratio by volume). The resultant bacterial solutions were then serially diluted in LB broth before mixing them with artificial sputum samples at the desired concentrations.

The experiments were performed by immersing cotton swabs in a solution of polystyrene and methyl cellulose with varying concentrations for $30 \mathrm{~s}$. The time $30 \mathrm{~s}$ have been optimised based on the real time swabbing techniques used in clinical procedures. The swabs were then air dried for $1 \mathrm{~min}$ and dipped in $700 \mu \mathrm{L}$ of DI water solution containing magnetic particles for elution. The motor was turned on with a specific set speed and the swabs were eluted for the desired amount of time. 


\section{Experimental Methods}

\subsection{Experimental Process}

In order to demonstrate the increase in elution from the swab due to impingement of the magnetic particles, experiments were performed in settings with and without magnetic agitation, using swabs that were dipped in artificial sputum samples containing polystyrene beads. In these experiments, dry swabs were immersed in the artificial sputum samples with $10 \% \mathrm{v} / \mathrm{v}\left(\sim 10^{9}\right.$ particles $\left./ \mathrm{mL}\right)$ polystyrene bead concentrations. Next, the swabs were air dried for $30 \mathrm{~s}$ and dipped into the vial containing DI water. The experiments were repeated $(n=5)$ for various elution times (30 s-180 s) at $30 \mathrm{~s}$ intervals. In another set of experiments $(n=5)$, swabs dipped in the same samples were dipped into the vial with DI water and were agitated using mechanical impingement caused by magnetic particles under the influence of the external magnet rotating at 300 RPM for various elution times (30 s-180 s). Recovery efficiencies were obtained by measuring the number of beads eluted into the DI water solution using techniques described in the methods section.

Rotational speed of the magnetic particles is an important parameter for elution, which determines the number of impacts to the swab fibres and therefore is related to the amount of material scrubbed per minute. In order to characterize its effect of rotational speed of the magnets on the number of eluted particles and recovery efficiency, dry swabs were immersed in artificial sputum solutions of $10 \%$ $\mathrm{v} / \mathrm{v}\left(\sim 10^{9}\right.$ polystyrene particles $\left./ \mathrm{mL}\right)$, air dried for $30 \mathrm{~s}$, immersed inside the vial with elution fluid, and exposed to various rotational speeds (0-500 RPM), for $30 \mathrm{~s}$. The number of eluted particles and recovery efficiencies were both recorded for five experiments at each rotational speed. The duration of elution procedure also has a significant effect on the recovery efficiency, since it determines the number of impingements that are caused over the entire period of time. To predict the nature of elution rate with changes in time, experiments were done with 10\% polystyrene in artificial sputum samples. Dry swabs were dipped in artificial sputum samples concentrated with $10 \% \mathrm{v} / \mathrm{v}\left(\sim 10^{9}\right.$ particles $/ \mathrm{mL}$ concentration) polystyrene beads, air dried for $30 \mathrm{~s}$ and immersed in the vial with elution fluid. The motor was set to a specific rotational speed and the number of eluted particles was noted with the change of time (30 s-120 s).

Experiments were also done with a specific elution time and rotational speed to determine the effects of concentration on recovery efficiency. The concentration of the sample also influences the recovery and is a vital parameter when body fluids with varying microbial concentrations are under consideration. Since it had already been established that higher rotational speeds (400-500 RPM) and elution times (120 s) produce a better recovery of beads, experiments were performed only with those specific parameters. Dry swabs were dipped in artificial sputum solutions of different concentrations $\left(\sim 10^{9}-10^{6}\right.$ particles $\left./ \mathrm{mL}\right)$, air dried for $30 \mathrm{~s}$ and immersed in DI water in a vial for elution. The rotational speed of the magnet was set at 400 or 500 RPM and the elution of materials from the swab was measured. Similar experiments were repeated with bacterial samples to show the respective changes in recovery efficiency with changes in rotational speed, time and concentration.

\subsection{Determination of Cellular Recovery}

The number of particles adhered to the swab before elution, were determined by measuring the volume of solution absorbed in the fibres, under the assumption that they were homogeneously mixed with the artificial sputum. To calculate the number of eluted particles, microscopic images (Upright Bright Field \& Fluorescence Microscope, Olympus, BX53, Tokyo, Japan) of $10 \mu \mathrm{L}$ of the post-elution solution on a microscope slide, covered by a slide cover $\left(22 \mathrm{~mm}^{2}\right)$, were taken at several points on the cover. These pictures were then assessed in ImageJ software (version 3.1.0) to automatically count the number of particles at each spots of the microscope slide cover. They were then averaged over the entire area to find the number of particles in the region that were covered by the slide cover and 
back-calculate to obtain the actual number of eluted particles in the solution. Recovery efficiency was calculated from the formula:

$$
\text { Recovery efficiency }=\frac{\text { No. of particles } / \text { cells in eluate solution after elution }}{\text { No. of particles/cells absorbed by swab with the sample before elution }}
$$

\subsection{Determination of Cellular Recovery for Low Concentration}

With a lower concentration, direct particle counting approach was not suitable, as the random sampling at a number of different locations led to a wider distribution in the number of particles at all locations. To overcome this problem, fluorescence intensity measurements were taken with a plate reader (Tecan Infinite M1000 Pro, Tecan Trading AG, Männedorf, Switzerland) of serially diluted samples of known concentration $(300 \mu \mathrm{L})$, and calibration curves were drawn. Separate calibration curves were drawn for E. coli and polystyrene beads to work with samples in $10^{6}-10^{4} \mathrm{CFU}$ or particles $/ \mathrm{mL}$ concentration range. Fluorescent intensities were measured for all of the post-elution solutions and compared with the calibration curve to know the exact concentrations. Once the elution volume has been measured, the number of recovered particles/cells were calculated by using the calibrated equation.

\subsection{Scanning Electron Microscopy (SEM) Measurements}

For a better understanding of the release of beads from swab fibres in a working model of polystyrene beads suspended in artificial mucous, Scanning Electron Microscopy (SEM) pictures were taken. SEM images of the swab tips were taken in order to visualize the elution of the microscopic particles from the swab head. Dry swabs were immersed in a solution of artificial sputum with a bead concentration of $10^{9}$ particles $/ \mathrm{mL}$ for $30 \mathrm{~s}$ and air dried for $30 \mathrm{~s}$. Some of these swabs were then introduced in the setup, and were eluted for $60 \mathrm{~s}$ in distilled water at 100 RPM. Others were preserved as is to obtain images of the pre-elution condition of the swab. Scanning Electron photomicrographs were then taken at magnifications of $1000 \times$ and $5000 \times$.

\section{Results}

\subsection{Effect of Magnetic Impingement on Elution}

Several experiments were done with the artificial samples containing polystyrene beads, as described in Section 3.1, and the resulting recovery efficiencies were recorded. The recovery efficiencies, as shown in Figure 3, increases with elution time, till $120 \mathrm{~s}$, and then remain steady. In the case of elution without agitation, the recovery efficiency increases from 33.68 to $41.8 \%$ when dipped in the DI water between 30 and $120 \mathrm{~s}$. Similarly, in the case of elution with magnetic agitation at 300 RPM, the recovery efficiency increases from $51 \%$ to $63.2 \%$ over $30-120 \mathrm{~s}$ of agitation. The difference in elution efficiency between agitated and non-agitated case was found to be statistically significant $\left(p\right.$-value $\left.<10^{-5}\right)$.

The capture and release of polystyrene beads has been demonstrated through scanning electron microscope images in Figure $4 \mathrm{a}$,b. The structural details of the fibres and the entrapment of polystyrene beads have been clarified with figures at $5000 \times$ magnifications (Figure $4 a, b$ ). Figure 4a shows the polystyrene beads retained in the swab fibres, when eluted without agitation, in close detail. The polystyrene beads are entrapped in the fibre matrix, and some beads also penetrate the inner matrix, when the swab was dipped in the sample. After elution without agitation, the artificial sputum on the top fibres dissolve and most of the particles on the top matrix are eluted. But, some of the fibres in the inner matrices are retained. Figure $4 \mathrm{~b}$ shows the swab fibres after elution by magnetic actuation. This figure shows that less number of beads are retained in the inner matrix of the swab as compared to the elution without agitation. Beads trapped in the small cracks and crevices are hard to remove, although the ones on the surface of fibres get eluted easily. Finally, these photographs also show the sources of the entrapment of beads, which cannot be removed, even with an active mechanism and higher impact force, thereby causing a deviation from $100 \%$ recovery. 


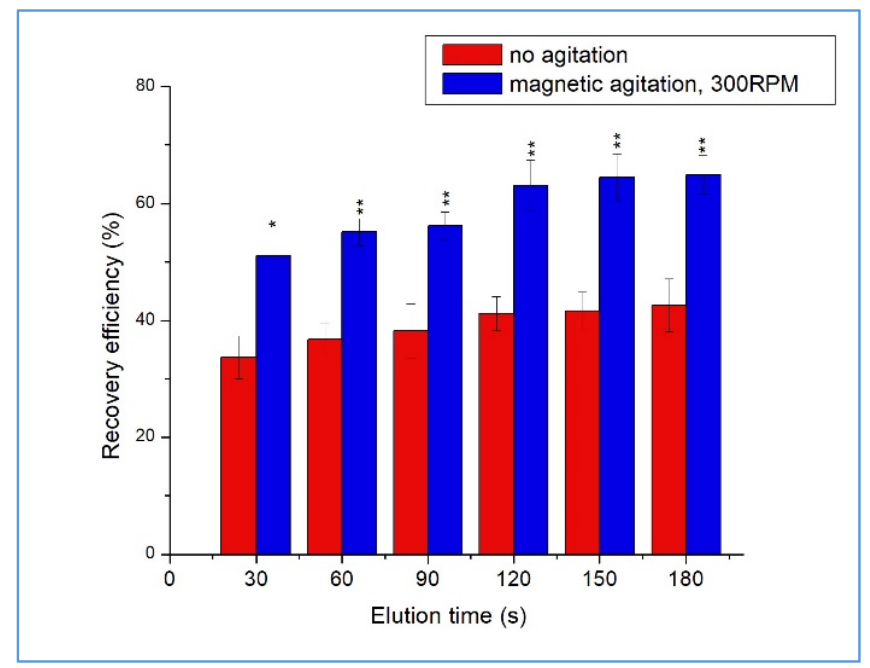

Figure 3. Comparison of recovery efficiencies for elution without agitation and with magnetic impingement (300 RPM) of swabs dipped in 10\% v/v $\left(\sim 10^{9}\right.$ particles $\left./ \mathrm{mL}\right)$ concentrated samples ( ${ }^{*}$ signifies $p>0.001,{ }^{* *}$ signifies $p<0.001$ ).

For better understanding, microscopic images were taken post elution at different concentrations (Figure $4 \mathrm{c}-\mathrm{f}$ ). $10 \mu \mathrm{L}$ of the post-eluted sample was taken on a microscopic plate, covered with a microscopic slide, and imaged under the fluorescent microscope. Higher number of particles are eluted when the initial concentration of the sample is higher. This is visible from the pictures, which were taken with different pre-elution concentrations from $1 \times 10^{6}$ to $1 \times 10^{9}$ particles $/ \mathrm{mL}$.
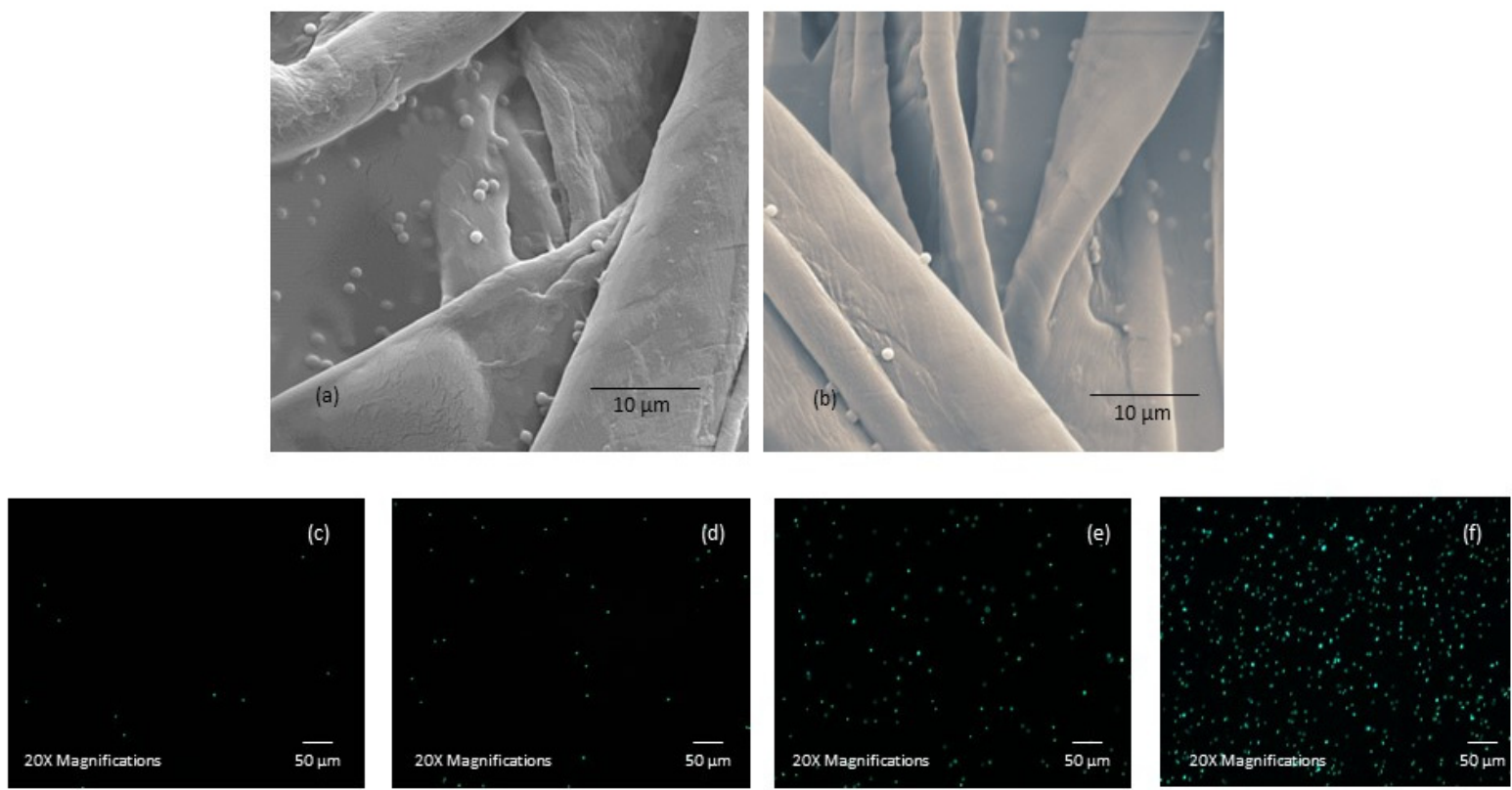

Figure 4. Distribution of polystyrene beads on swab fibres (a) post elution (no agitation); (b) post elution (magnetic beads agitation) $-5000 \times$. Fluorescent microscopic images of post elution at different bead concentration: (c) $10^{6}$ particles $/ \mathrm{Ml}$; (d) $10^{7}$ particles $/ \mathrm{mL}$; (e) $10^{8}$ particles $/ \mathrm{mL}$; and, (f) $10^{9}$ particles $/ \mathrm{mL}$. 


\subsection{Characterization of Magnetically Induced Mechanical Impingement}

\subsubsection{Effect of Rotational Speed of the Magnet and Elution Time}

Several experiments were performed, according to the procedure shown in Section 3.1, to examine the change in recovery efficiencies with varying rotational speed and elution time. Each dry swab weighing $0.41 \pm 0.07 \mathrm{~g}$ absorbs an average of $227 \pm 28 \mu \mathrm{L}$ of the sample when dipped into artificial sputum matrix loaded with particles $\left(1 \times 10^{9}\right.$ particles $\left./ \mathrm{mL}\right)$. Therefore, the number of particles that are absorbed in the swab was calculated to be $(1.8 \pm 0.3) \times 10^{8}$ particles $/ \mathrm{mL}$. Upon elution with magnetic agitation, the number of particles eluted increased with the rotational speed. At lower rotational speed of $100 \mathrm{RPM}$, the number of eluted particles were $\sim 4 \times 10^{7}$ but it increased rapidly to about $\sim 7 \times 10^{7}$ particles at 500 RPM, when the elution time was increased to $60 \mathrm{~s}$, the number of eluted particles increased for each rotational speed. The total number of eluted particles increased to $\sim 6 \times 10^{7}$ at $100 \mathrm{RPM}$ and it gradually rose to $\sim 8 \times 10^{7}$ at a higher speed (500 RPM). The corresponding change in recovery efficiency with rotational speed at same bead concentration $\left(\sim 10^{9}\right.$ particles $\left./ \mathrm{mL}\right)$ is shown in Figure 5a. At lower elution time (30 s) and speed (100 RPM), the recovery efficiency (38.4\%) was slightly better than the case with no-agitation (33.7\%). But, with an increase in rotational speed, the recovery efficiency increased to $67.6 \%$ at 500 RPM, and then remained constant.

With higher elution times, the number of eluted particles increase. At 90 s elution time, the total number of eluted particles increased from $\sim 6.5-10 \times 10^{7}$ within 100-500 RPM and then remained constant. Similarly, at 120 s elution time, the corresponding values are $\sim 8 \times 10^{7}-10^{8}$ within the same speed range. The corresponding recovery efficiency increased for every rotational speed when the elution times were increased. The highest recovery efficiencies were found at $120 \mathrm{~s}$ elution time, where the values increased from $44.6 \%$ at 100 RPM to $73.4 \%$ at 500 RPM and then remained constant. In this case, also, the average results between 100 RPM and the method of no agitation is not significant ( $p$-value $=0.14)$, but becomes significant at 300 RPM ( $p$-value $=0.0007)$.

The changes in recovery efficiency with time for elution with a sample of $10 \% \mathrm{v} / \mathrm{v}$ $\left(\sim 10^{9}\right.$ particles $\left./ \mathrm{mL}\right)$ concentration is shown in Figure $5 \mathrm{~b}$. The recovery efficiency increased with the elution time. In the control case, without magnetic actuation, there was a small increase in recovery with elution time (33.7-41\% within $30 \mathrm{~s}-150 \mathrm{~s})$. At low rotational speed (100 RPM), the recovery efficiency increased from $39.3 \%$ at $30 \mathrm{~s}$ to $48.6 \%$ at $120 \mathrm{~s}$, and then remained steady. Higher elution time resulted in an increased efficiency, but with a less significant rate of increase than the effects that were seen with rotational speed. As shown previously, the recovery efficiency increases with the rotational speed. But at higher speeds, the recovery efficiencies also attain a steady value much faster. For example, at 400 RPM rotational speed, it remains horizontal at around 62\%. At 500 RPM, the recovery efficiencies increase from $67.9 \%$ at $30 \mathrm{~s}$ to $71.2 \%$ at $90 \mathrm{~s}$, and became constant afterwards. The effects of elution time on recovery efficiency is significant at lower speeds as compared to higher speeds. With lower rotational speeds (100 RPM), the recovery efficiency shows an increase of $9 \%(39 \%$ to $48 \%$ ) over elution time (30 s-150 s). But, at higher rotational speeds, the slope of the curve decreases (only about $4 \%$ increase in recovery efficiency) and elution time does not have a pronounced effect on time. When considering both of these parameters, an operating condition of 500 RPM and $120 \mathrm{~s}$ are found to be suitable for maximum recovery. Even though there is a very low increase in average efficiency, the recovery efficiency values are not significant between $30 \mathrm{~s}$ and $60 \mathrm{~s}(p$-value $=0.56)$ and between $30 \mathrm{~s}$ and $120 \mathrm{~s}$ ( $p$-value $=0.21$ ) of elution time. 


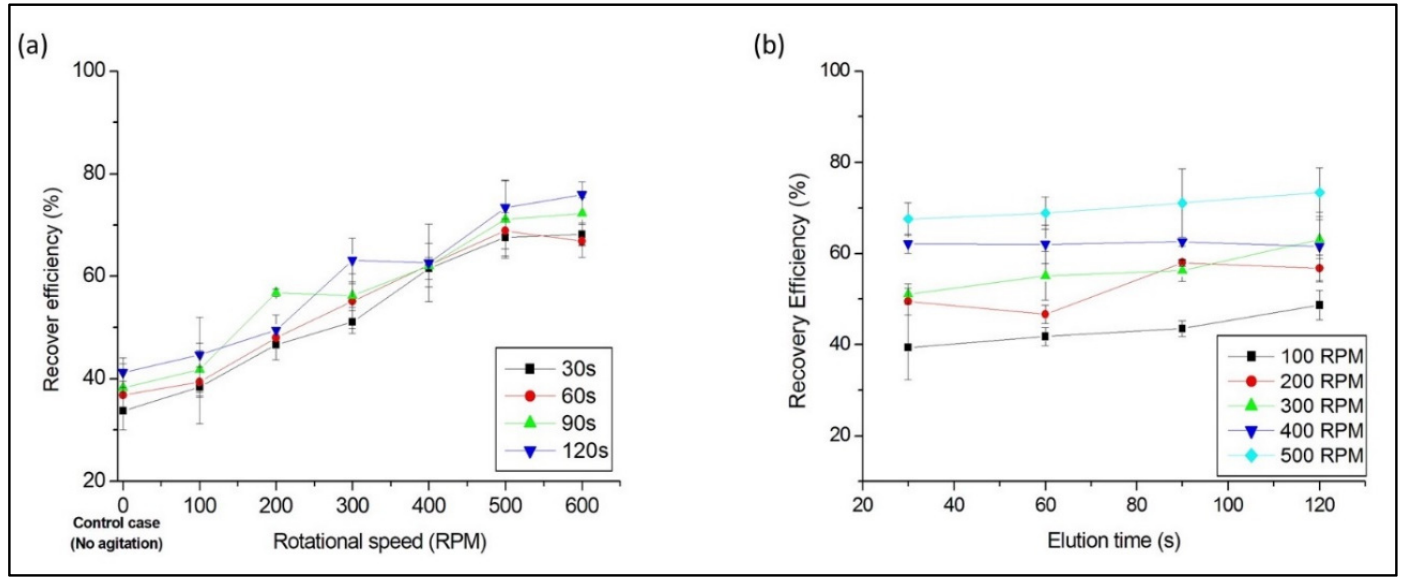

Figure 5. (a) Effect of rotational speed (RPM) on the recovery efficiency of polystyrene beads $\left(10^{9}\right.$ particles $\left./ \mathrm{mL}\right)$; (b) Effect of time on Recovery efficiency $\left(10^{9}\right.$ particles $\left./ \mathrm{mL}\right)$.

\subsubsection{Effect of Concentration}

Figure 6 shows the recovery efficiency that was obtained at various concentrations of the sample at these rotational speeds. From it can be seen from the result that higher recovery efficiencies are obtained at lower concentrations. At $0.01 \% \mathrm{v} / \mathrm{v}$ concentration $\left(10^{6}\right.$ particles $\left./ \mathrm{mL}\right)$ of particles in artificial sputum solution, the efficiency reaches to $92.38 \%$ at 500 RPM and $89.84 \%$ at 400 RPM. Higher concentrations $\left(10 \% \mathrm{v} / \mathrm{v}\right.$ concentration, $1 \times 10^{9}$ particles $\left./ \mathrm{mL}\right)$ yield lower recovery $(61.57 \%$ and $72.2 \%$, respectively, for 400 and 500 RPMs, respectively).

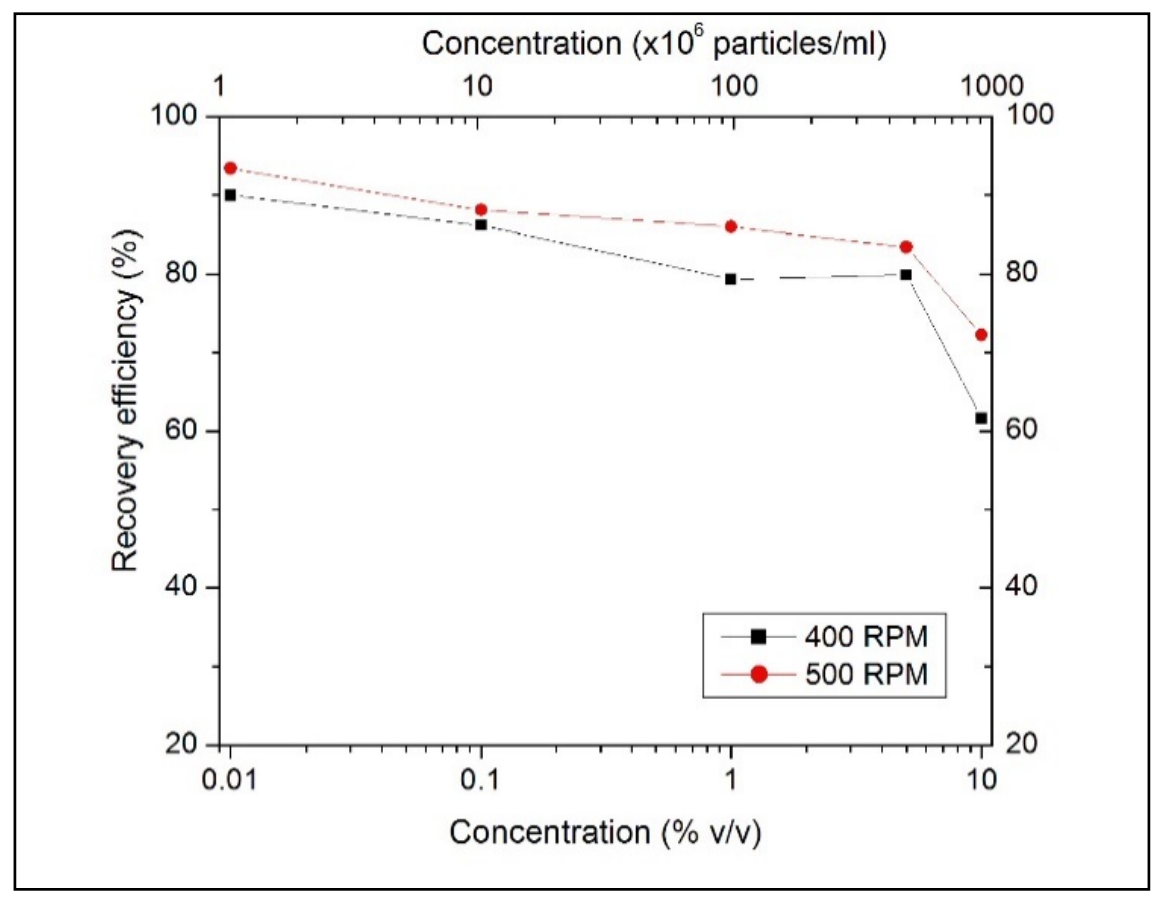

Figure 6. Effect of concentration of beads on the recovery efficiency (120 s, 400-500 RPM). 


\subsection{Bacterial Samples}

\section{Effect of Rotational Speed and Concentration}

Similar experiments were done with cultured E. coli mixed with the artificial mucous at various concentrations. The swab was dipped in the sample, air dried for $30 \mathrm{~s}$, and eluted in DI water for $2 \mathrm{~min}$ at different rotational speeds. The recovery efficiency obtained at various rotational speed is shown in Figure 7. They show that the recovery efficiency increases with rotational speed initially and gradually plateaus after 500 RPM. Recovery efficiencies close to $85 \%$ are obtained at 500 RPM, while they are low at around $47 \%$ at 100 RPM. There was no statistical difference in the results that were obtained between $6.4 \times 10^{9}$ and $6.3 \times 10^{8} \mathrm{CFU} / \mathrm{mL}$ samples.

Elution with lower concentrations of E. coli in the sample show a similar increasing trend in recovery efficiency (Figure 7). For $\sim 2 \times 10^{6} \mathrm{CFU} / \mathrm{mL}$ concentration, the average number of eluted particles range from $\sim 1-1.5 \times 10^{6} \mathrm{CFU} / \mathrm{mL}$ over 100-500 RPM. This corresponds to a recovery increase from $49.1 \%$ to $86 \%$, with an average error of $\pm 3.4 \%$. For $\sim 4 \times 10^{5} \mathrm{CFU} / \mathrm{mL}$ samples, the efficiencies increase from $50.8 \%$ to $86.6 \%$ over the same range of rotational speed, and almost coincides with the values of the previous curve for higher rotational speeds. Higher recovery $(90.42 \%)$ can be obtained at $500 \mathrm{RPM}$ with $3.3 \times 10^{5} \mathrm{CFU} / \mathrm{mL}$ sample and also corresponds to a steeper slope than the sample with $\sim 1 \times 10^{6} \mathrm{CFU} / \mathrm{mL}$ concentration Similar to the previous experiment with polystyrene beads, the average recovery efficiencies between the control case and that of different speeds are insignificant at 100 RPM ( $p$-value $=0.3)$, but is statistically significant at 300 RPM ( $p$-value $=0.006$ ). Also, when compared between two different concentrations, the recovery efficiency values between control case of no agitation and lower rotational speeds are insignificant at $100 \mathrm{RPM}$ ( $p$-value $=0.41$ ), but the values are close to statistically significance at higher rotational speeds ( $p$-value $=0.067$ at 400 RPM). As seen in the case of polystyrene beads, at higher rotational speeds (400 and 500 RPM), there is a significant increase in efficiency with lower concentrations than that at a higher concentration. Hence, working with a lower concentration of cells can be beneficial at higher rotational speeds.

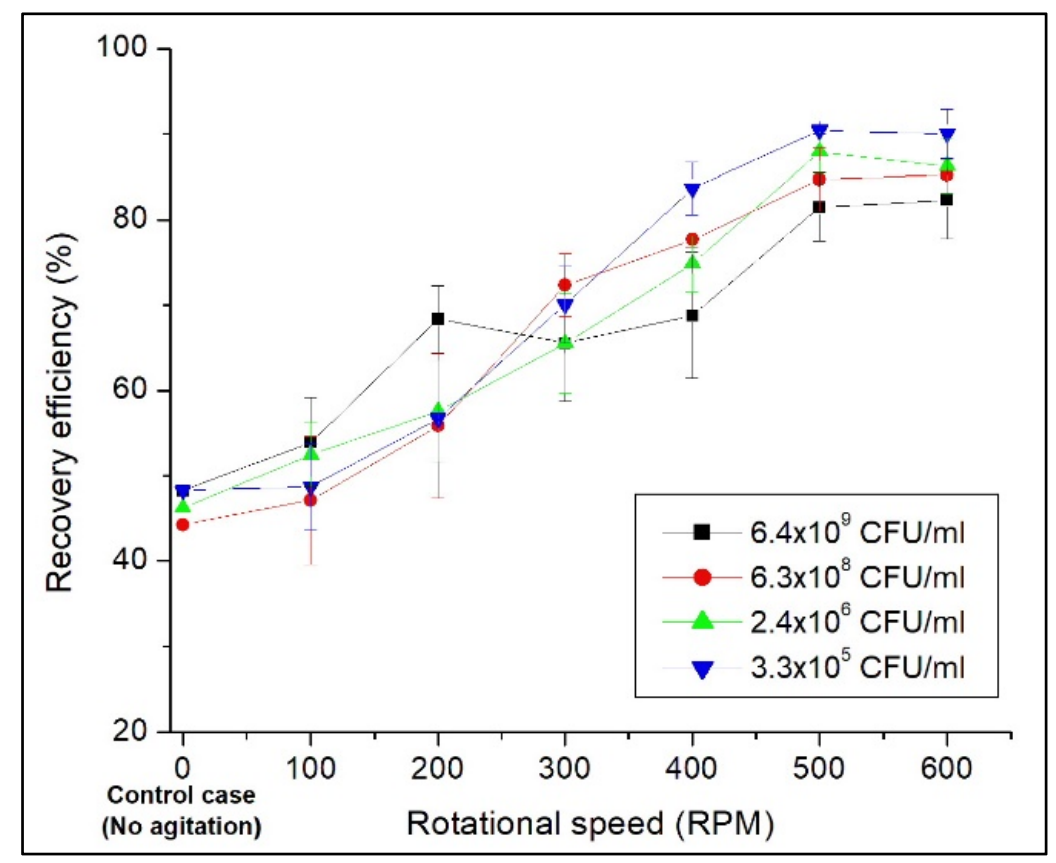

Figure 7. Effect of RPM on recovery of E. coli samples at different concentrations, 120 s elution time. 


\section{Discussion}

Elution by magnetic agitation has been proved to be beneficial over the control case of no agitation, as shown in Figure 3. Two different mechanisms operate during the processes of elution by magnetic agitation. The magnetic impingement on the swab fibres causes the sample, absorbed in the swab, to be locally squeezed out at the point of impingement. This eluted sample is thoroughly mixed with the elution buffer due to the convective motion of the surrounding fluid, which is also caused by the motion of the magnets. Higher recovery efficiencies are obtained from physical impingement of particles on the swab tip, and hence is a better elution process than any technique without significant agitation. Unlike the no agitation case, where the time constant that is associated with the dissolution process is long and therefore only the sample at the surface layers of the swab get effectively eluted, under magnetic impingement, not only is the surface elution rapid, but the impingement also imparts forces to actively mix the sputum sample between the deeper layers of the swab and the surface layers that have absorbed the elution buffer from outside. This effectively helps in the dissolution of the sample that has been absorbed deep inside the swab matrix, which may not be eluted otherwise.

The recovery efficiency of polystyrene beads from the swab fibres increases with an increase in rotational speed, as shown in Figure 5a. Multiple impingements on the swab fibres are responsible for these higher rates of elution. The magnetic particles rotate at $2 \times i \times N_{m}$, where $N_{m}$ is the rotational speed of the motor and $i$ is the gear ratio. In one complete rotation, the magnetic particles impart two impacts. Hence, the total number of impacts per minute becomes $4 \times i \times N_{m}$. As the rotational speed increases, the rate of impact on the swab fibres increases. The number of impacts at 500 RPM is 5 times higher than that offered at a low rotational speed of 100 RPM. Higher forces of impact between the particles and the swab provide better release of the samples due to efficient scrubbing action. Fresh elution fluid enters the fibre matrix after each subsequent impact and allows for the faster dissolution of artificial sputum. More particles can migrate into the elution volume, facilitating better recovery, and hence more beads are eluted from the swab tip. The acting forces on the swab were measured using a load cell, as shown in Figure $2 b$. The impact forces was found to increase with the increase in the number of impacts per minute at higher rotational speeds. The forces on the swab are mostly due to impact of the rotating magnetic particles, although the whole kinetic energy is not imparted to the swabs. A part of the kinetic energy is also dissipated in the fluid, which causes convective fluid motion around the swab. The duration of elution also determines the number of impingements that are caused. When the rotational speeds are kept constant, the total number of impacts per minute remains the same over the entire time period. At lower elution times, the total number of impacts $\left(4 \times i \times N_{m} \times t\right)$ has a significant effect on the number of eluted beads. For 30, 60 and $90 \mathrm{~s}$, the increase in total number of impacts over time results in increased average impact force on the swab fibres, thereby increasing the number of recovered particles and the efficiency. Fresh fluid entering the fibre matrix helps in dissolving the artificial sputum solutions that pushes the beads into the elution fluid. But, the dissolution reaches a steady state after a very small amount of time, and after that, the impact is the only operating force that is acting on the swab. The impact force that is surrounding the swab removes the beads from the swab matrix, but only to a certain extent. After $\sim 90 \mathrm{~s}$, most of the beads that were free to move to the eluate had already migrated. The ones trapped deeper into the fibres do not get eluted and remains inside, even at higher elution times.

The increase in recovery efficiency at a lower concentration (as shown in Figure 6) is a surprising result and it could be due to a varied number of factors. The ability of the artificial sputum sample to dissolve into the eluent fluid could be influenced by the particle loading. High particle loading could make it more difficult to dissolve samples that are embedded in the deeper layers of the swab and therefore produce lower elution efficiency. Alternatively, the process of reabsorption of the eluent fluid after local impact into the swab and the particle loading of the surface layers due to the reabsorption can be influenced by the concentration of the particles in solution. In low concentration samples, the number of particles in the eluent solution is comparatively low. The reabsorption could preferentially absorb some of the fluid post elution and therefore retain more of the particles in the 
eluent fluid as compared to the high concentration case. Finally, after the elution process is complete, some of the fluid is adsorbed on the outer surface of the swab while it is being taken out, which is also directly proportional to the concentration and the number of available adsorption sites.

E.coli was chosen as a model as it is one of the common infections in many clinical situations. It is also similar in size to many other infectious pathogens. Additionally, it is easy to grow in laboratory conditions, and therefore was considered as a suitable model. The results with E.coli showed a similar trend as the polystyrene beads indicating that the characterization performed with the beads would be useful in determining the optimal conditions for bacterial samples as well. Similar to the polystyrene beads, the bacterial cells also undergo higher rates of recovery efficiency at higher speeds, as shown in Figure 7 . The recovery efficiency was found to increase with concentration of the bacteria in the control case (no agitation). This is associated with the dissolution of the surface layer of artificial sputum dissolving into the eluent solution. In all of the other cases, the impingement of the magnetic particles on the swab creates impact forces that physically squeeze the fluid from the fibers and push the sample out, which results in higher elution efficiency.

\section{Conclusions}

A mechanical technique of elution of viscous sample from cotton swab fibres has been developed where the mechanical impingement is imposed by magnetic particles under the influence of a rotating magnetic field. The rotating magnetic field caused not only impingement of the magnetic particles on the swab, but also recirculation of the elution fluid, thereby enhancing the elution process. A device was designed, developed, and characterized using fluorescent polystyrene beads. The elution efficiency was also found to be related to the time of exposure to mechanical impingement and an optimal time of $120 \mathrm{~s}$ was determined. Next, the optimized device was also tested on artificial sputum samples that were loaded with $E$. coli bacteria. The elution efficiency was found to be linked to the rotational speed of the magnet and efficiencies as high as 90\% was obtained at 500 RPM. This method of elution has broad applicability in a variety of sample pre-processing procedures, both in biological analysis and clinical diagnostics.

Acknowledgments: The authors acknowledge support from the Natural Science and Research Council (NSERC) and the Canadian Institute of Health Research (CIHR) of Canada through the Collaborative Health Research Program (CHRP). P.R.S also acknowledges support from the Canada Research Chairs Program.

Author Contributions: Shubham Banik and P. Ravi Selvaganapathy conceived and designed the experiments; Shubham Banik performed the experiments; Shubham Banik and P. Ravi Selvaganapathy analyzed the data; P. Ravi Selvaganapathy and James Mahony contributed reagents and materials; Shubham Banik, James Mahony and P. Ravi Selvaganapathy wrote the paper.

Conflicts of Interest: The authors declare no conflict of interest.

\section{References}

1. Pang, B.C.M.; Cheung, B.K.K. Double swab technique for collecting touched evidence. Leg. Med. 2007, 9, 181-184. [CrossRef] [PubMed]

2. Hodges, L.R.; Rose, L.J.; O'Connell, H.; Arduino, M.J. National validation study of a swab protocol for the recovery of Bacillus anthracis spores from surfaces. J. Microbiol. Methods 2010, 81, 141-146. [CrossRef] [PubMed]

3. Rose, L.; Jensen, B.; Peterson, A.; Banerjee, S.N.; Arduino, M.J. Swab materials and Bacillus anthracis spore recovery from nonporous surfaces. Emerg. Infect. Dis. 2004, 10, 1023-1029. [CrossRef] [PubMed]

4. Stricklin, S.N. The Evaluation of Different Collection Methods for the Optimum Recovery of DNA from Bloodstains on Various Surfaces; University of North Texas Health Science Center at Fort Worth: Fort Worth, TX, USA, 2013.

5. Chastre, J.; Fagon, J.Y.; Bornet-Lecso, M.; Calvat, S.; Dombret, M.C.; al Khani, R.; Basset, F.; Gibert, C. Evaluation of bronchoscopic technique for the diagnosis of nosocomial pneumonia. Am. J. Respir. Crit. Care Med. 1995, 152, 231-240. [CrossRef] [PubMed]

6. Sahin, S.; Saygun, I.; Kubar, A.; Slots, J. Periodontitis lesions are the main source of salivary cytomegalovirus. Oral Microbiol. Immunol. 2009, 24, 340-342. [CrossRef] [PubMed] 
7. Najar, M.; Saldanha, C.; Banday, K. Approach to urinary tract infections. Indian J. Nephrol. 2009, 19, 129. [CrossRef] [PubMed]

8. Voorhees, J.C.; Ferrance, J.P.; Landers, J.P. Enhanced elution of sperm from cotton swabs via enzymatic digestion for rape kit analysis. J. Forensic Sci. 2006, 51, 574-579. [CrossRef] [PubMed]

9. Norris, J.V.; Manning, K.; Linke, S.J.; Ferrance, J.P.; Landers, J.P. Expedited, chemically enhanced sperm cell recovery from cotton swabs for rape kit analysis. J. Forensic Sci. 2007, 52, 800-805. [CrossRef] [PubMed]

10. Hulme, P.; Lewis, J.; Davidson, G. Sperm elution: An improved two phase recovery method for sexual assault samples. Sci. Justice 2013, 53, 28-33. [CrossRef] [PubMed]

11. Lutz, J.K.; Crawford, J.; Hoet, A.E.; Wilkins, J.R.; Lee, J. Comparative performance of contact plates, electrostatic wipes, swabs and a novel sampling device for the detection of Staphylococcus aureus on environmental surfaces. J. Appl. Microbiol. 2013, 115, 171-178. [CrossRef] [PubMed]

12. Dauphin, L.A.; Marston, C.K.; Bhullar, V.; Baker, D.; Rahman, M.; Hossain, M.J.; Chakraborty, A.; Khan, S.U.; Hoffmaster, A.R. Swab protocol for rapid laboratory diagnosis of cutaneous anthrax. J. Clin. Microbiol. 2012, 50, 3960-3967. [CrossRef] [PubMed]

13. Walker, R.E.; Petersen, J.M.; Stephens, K.W.; Dauphin, L.A. Optimal swab processing recovery method for detection of bioterrorism-related Francisella tularensis by real-time PCR. J. Microbiol. Methods 2010, 83, $42-47$. [CrossRef] [PubMed]

14. Brown, G.S.; Betty, R.G.; Brockmann, J.E.; Lucero, D.A.; Souza, C.A.; Walsh, K.S.; Boucher, R.M.; Tezak, M.S.; Wilson, M.C.; Rudolph, T.; et al. Evaluation of rayon swab surface sample collection method for Bacillus spores from nonporous surfaces. J. Appl. Microbiol. 2007, 103, 1074-1080. [CrossRef] [PubMed]

15. Elutions Prior to Antibody Identification. Available online: https://sites.ualberta.ca/ pletendr/tmmodules/methods /70met-elute-ab.html (accessed on 28 February 2017).

16. Kuttath, V.; George, B.; Sudhakar, S. Removal of Antibodies from Direct Antiglobulin Test Positive Red Blood Cells. Acad. Med. J. India 2015, III, 51-54.

17. Wuske, T.; Polzius, R.; Mahn, J.; da Costa, M.C. Swab Analyzer for the Immunochemical Detection of Substances. U.S. Patent No. 6,375,896, 23 April 2002.

18. Pelssers, E.G.M.; Nieuwenhuis, J.H. Sampling Device and Sampling Method. U.S. Patent No. 8,770,049, 8 July 2014.

19. Fabra, J.C.; Gibert, R.B.; Casas, A.C. Swab Elution Chamber in a Test Cartridge. U.S. Patent No. 9,063,037, 11 July 2013.

20. Nason, F.L. Specimen Test Unit. U.S. Patent No. 4,978,504, 30 November 1993.

21. Dryga, S.A.; Esch, V.C.; Saul, R.G.; McDowell, A.F. Separating Target Analytes Using Alternating Magnetic Fields. U.S. Patent No. 9,389,225, 27 October 2011.

22. Allard, J.E.; Baird, A.; Davidson, G.; Jones, S.; Lewis, J.; McKenna, L.; Weston, C.; Scrimger, D.; Teppett, G. A comparison of methods used in the UK and Ireland for the extraction and detection of semen on swabs and cloth samples. Sci. Justice 2007, 47, 160-167. [CrossRef] [PubMed]

23. Sigma-Aldrich. Product Information: Methtyl Cellulose. 1997. Available online: https://www.sigmaaldrich. com/content/dam/sigma-aldrich/docs/Sigma-Aldrich/Product_Information_Sheet/m0262pis.pdf (accessed on 29 August 2017).

24. Lopez-Vidriero, M.T.; Charman, J.; Keal, E.; De Silva, D.J.; Reid, L. Sputum viscosity: Correlation with chemical and clinical features in chronic bronchitis. Thorax 1973, 28, 401-408. [CrossRef] [PubMed]

(C) 2017 by the authors. Licensee MDPI, Basel, Switzerland. This article is an open access article distributed under the terms and conditions of the Creative Commons Attribution (CC BY) license (http:/ / creativecommons.org/licenses/by/4.0/). 